\title{
Keratan Sulfate Glycosaminoglycan and the Association with Collagen Fibrils in Rudimentary Lamellae in the Developing Avian Cornea
}

\author{
Robert D. Young, ${ }^{1}$ E. Claire Gealy, ${ }^{2}$ Melody Liles, ${ }^{1}$ Bruce Caterson, ${ }^{2}$ James R. Ralphs, ${ }^{2}$ and \\ Andrew J. Quantock ${ }^{1}$
}

\begin{abstract}
Purpose. Keratan sulfate (KS), through its association with fibrillar collagen as KS-substituted proteoglycan (KS PG), is thought to be instrumental in the structural development of the corneal stroma. The authors used two different sulfate motif-specific antibodies to identify the sequence of appearance, and the association with collagen, of sulfated KS during avian corneal morphogenesis.
\end{abstract}

Methods. Corneas from chicken embryos throughout the developmental period, from day 8 through day 18 of incubation, were examined by immunofluorescence and immunoelectron microscopy using monoclonal antibodies 5D4 and 1B4, which react with high- and low-sulfated epitopes on KS, respectively.

Results. KS was identified as punctate labeling at incubation day 8 , the earliest stage examined, suggesting a cell-associated distribution. By day 10, labeling was more homogeneous, indicating that KS sulfation motifs were present in the stromal extracellular matrix. At day 12 through day 14, immunopositive sites were concentrated primarily in the anterior stroma but became more uniform throughout the full stromal thickness by day 18 . From day 10 on, electron microscopy revealed a high-sulfated KS epitope closely associated with bundles of regularly arranged collagen fibrils, initially near cell surfaces in rudimentary lamellae. Individual cells, associated with collagen bundles with different fibril orientations, imply the potential for simultaneous deposition of multiple lamellae.

Conclusions. During chick corneal morphogenesis, significant matrix deposition of high-sulfated KS epitope occurs by day 10, with accumulation subsequently proceeding in an anterior-toposterior manner. High-sulfated KS likely serves to help define the regular spatial organization of collagen fibrils in bundles newly extruded into the extracellular milieu. (Invest Ophthalmol Vis Sci. 2007;48:3083-3088) DOI:10.1167/iovs.06-1323

Corneal morphogenesis in the chick is achieved through a series of well-defined developmental events. Formation of the corneal stroma starts with the deposition of a loose primary

From the ${ }^{1}$ Structural Biophysics Group, School of Optometry and Vision Sciences, and the ${ }^{2}$ Connective Tissue Biology Laboratory, School of Biosciences, Cardiff University, Cardiff, United Kingdom.

Supported by Biotechnology and Biological Sciences Research Council Project Grant BBS/B/10994 (AJQ, BC, JRR)

Submitted for publication November 3, 2006; revised February 16, 2007; accepted May 3, 2007.

Disclosure: R.D. Young, None; E.C. Gealy, None; M. Liles, None; B. Caterson, None; J.R. Ralphs, None; A.J. Quantock, None Presented at the annual meeting of the Association for Research in Vision and Ophthalmology, Fort Lauderdale, Florida, April 2006.

The publication costs of this article were defrayed in part by page charge payment. This article must therefore be marked "advertisement" in accordance with 18 U.S.C. $\$ 1734$ solely to indicate this fact.

Corresponding author: Andrew J. Quantock, Structural Biophysics Group, School of Optometry and Vision Sciences, Cardiff University, Redwood Building, King Edward VII Avenue, Cardiff CF10 3NB, UK; quantockaj@cardiff.ac.uk. matrix of collagen fibrils by ectodermal cells, destined to become the corneal epithelium, at around day 3 of incubation. Mesenchymal cells of neural crest origin subsequently migrate inwardly along the surface of the lens, posterior to the primary stroma, and form the corneal endothelium by day 4 . The primary stroma then swells and by day 7 is invaded by a second wave of mesenchymal cells, the presumptive corneal fibroblasts, that synthesize and deposit the macromolecular components, collagens, and particularly the proteoglycans of the mature, or secondary, stroma. ${ }^{1-5}$

The manner in which collagen fibrils are laid down by corneal fibroblasts is key for tissue structure and function, particularly because light transmission and biomechanical stability are dependent on the establishment of a stacked lamellar array of regularly spaced, uniformly thin collagen fibrils. ${ }^{6-8}$ With the use of electron microscopy, Birk and Trelstad ${ }^{9}$ discovered that collagen fibrillogenesis in the developing chick cornea occurs initially in small recesses at the keratocyte cell surface, where small bundles of collagen fibrils are deposited. Subsequently, fibril bundles coalesce to form larger bundles, and eventually lamellae, so that cell alignment in the embryonic cornea most likely has a significant bearing on eventual tissue architecture.

Several lines of evidence implicate keratan sulfate (KS), the major glycosaminoglycan (GAG) of corneal stroma, as a regulator of corneal matrix architecture at the fibrillar level. It has been detected in biochemical and immunohistochemical analyses of the chick corneal stroma by day 6 , soon after the invasion of presumptive keratocytes. ${ }^{10-14}$ Its distribution remains mostly intracellular until day 9, suggesting a lag between the initiation of KS biosynthesis and the secretion of significant amounts into the extracellular matrix. ${ }^{12}$ Biosynthesis of KS then increases until day $14,{ }^{11,12}$ after which a number of chemical and physicochemical changes in GAG sulfation occur. ${ }^{10,11}$ mRNA for the major KS-containing PG, lumican, is present throughout development at higher levels than message for the other two KS PGs, keratocan and mimecan, with lumican likely bearing most of the KS GAGs. ${ }^{15}$

Recently, electrospray ionization tandem mass spectroscopy was used to obtain precise quantitation of sulfated KS disaccharides in enzyme digests of chick corneal sections between day 8 and 20 of development. ${ }^{16}$ However, to understand the sequence and spatial deposition of sulfated KS and to relate this to the assembly of an organized lamellar array of collagen fibrils in the stroma, detection and observation of KS in undisrupted tissue are required. For this purpose, we carried out an immunofluorescence and immunoelectron microscopic study using the sulfate motif-specific monoclonal antibodies 5D4 and 1B4 to localize sulfated KS PG epitopes in intact tissue sections. 5D4 recognizes linear sequences of $\mathrm{N}$-acetyl lactosamine disaccharides of KS PGs, with both GlcNAc and Gal sulfated and with a minimal epitope requirement of a pentasulfated hexasaccharide. 1B4 reacts with lesser sulfated sequences of N-acetyl lactosamine disaccharides. ${ }^{17,18}$ We could thus investigate the spatial location of high- and low-sulfated KS in relation to 
developmental time points to test the hypothesis that the appearance of sulfated KS is linked to the acquisition of lamellar organization of collagen fibrils as the stroma matures.

\section{Materials AND Methods}

Fertilized White Leghorn chicken eggs were incubated at $38^{\circ} \mathrm{C}$ in a humidified chamber. At 8, 10, 12, 14, 16, and 18 days of incubation, embryos were killed in accordance with the ARVO Statement for Use of Animals for Ophthalmic and Vision Research and local ethical rules. Corneas (or whole eyes at day 8) were then carefully excised and processed for microscopy.

\section{Immunofluorescence Microscopy}

Two to three corneal specimens at each stage from day 8 through day 18 were frozen unfixed on dry ice in OCT embedding medium. Serial $10-\mu \mathrm{m}$ cryosections were cut in the transverse plane and collected on adhesive, charged-surface slides (Histobond; Leica Microsystems, Nussloch, Germany). For immunolabeling, cryosections were rehydrated in PBST $(1 \times$ PBS containing $0.1 \%$ Tween-20) for 5 minutes and were blocked in 5\% normal goat serum (prepared in PBST). Cryosections were indirectly labeled with monoclonal antibodies 5-D-4 (which recognizes oversulfated $\mathrm{N}$-acetyl lactosamine disaccharides of KS) or 1-B-4 (which recognizes lesser sulfated $\mathrm{N}$-acetyl lactosamine disaccharides of KS) ${ }^{17,18}$ for 3 hours at room temperature using a 1:500 dilution of the antibody supernatants. Sections were washed in PBST and detected by incubation with Alexa Fluor $488 \mathrm{~F}\left(\mathrm{ab}^{\prime}\right)_{2}$ fragments of goat anti-mouse IgG (Molecular Probes, Invitrogen, Eugene, OR) at a concentration of $5 \mu \mathrm{g} / \mathrm{mL}$ for 1 hour at room temperature. Negative antibody controls were performed in each immunolabeling experiment where mouse immunoglobulin (MIgG) served as a primary antibody control and PBST as a secondary antibody control. Sections were washed in PBST and mounted in antifade medium (Vectashield; Vector Laboratories, Burlingame, CA) containing the nuclear counterstain 4',6 diamidino-2phenylindole (DAPI). Sections were analyzed under a microscope (BX61; Olympus, Tokyo, Japan), and images were recorded with a digital camera (F-View; Olympus Industrial America, Orangeburg, NY).

\section{Immunoelectron Microscopy}

Two to three corneas at each day of incubation, from day 8 to day 18 , were mildly fixed for 1 hour in $4 \%$ paraformaldehyde in $0.1 \mathrm{M}$ Sorensen phosphate buffer at $\mathrm{pH} 7.4$, dehydrated in $55 \%, 70 \%$, and $90 \%$ ethanol at $-15^{\circ} \mathrm{C},-20^{\circ} \mathrm{C}$, and $-25^{\circ} \mathrm{C}$, respectively, then embedded in Lowicryl $\mathrm{K} 4 \mathrm{M}$ resin, which was polymerized by ultraviolet light at $-25^{\circ} \mathrm{C}$. All procedures at low temperature were carried out in a freeze substitution unit (Reichert AFS; Leica UK, Milton Keynes, UK). Sections (approximately 100-nm thick) were cut with an ultramicrotome (UC6; Leica Microsystems GmbH, Wetzlar, Germany) and picked up on uncoated nickel grids, which were floated section-side down on $25-\mu \mathrm{L}$ droplets of immunoreagents, as follows: after 30 minutes on PBS containing $1 \%$ BSA, to block nonspecific binding, they were transferred to primary monoclonal antibodies $1 \mathrm{~B} 4$ or 5D4 diluted 1 in 100 for 2 hours in the same buffer. Sections were washed free of unbound antibody by flotation on several droplets of buffer alone over 30 minutes before antibody-positive sites were detected by floating grids for 1 hour on goat anti-mouse secondary antibody 10-nm gold conjugate, diluted 1 in 20 (BB International Ltd., Cardiff, UK). Nonspecific labeling of secondary antibody was assessed in sections in which the primary antibody was replaced with PBS/BSA alone. Sections were contrasted with uranyl acetate and lead citrate and were examined in a transmission electron microscope (FEI EM208; Philips, Hillsboro, OR) operating at $80 \mathrm{kV}$.

\section{Results}

Sulfated KS was identified by immunofluorescence microscopy in the developing chick cornea from day 8 of incubation through day 18 with the 5D4 (Figs. 1A-D) anti-KS monoclonal antibody. Labeling appeared punctate at the earliest stages examined but became more homogeneous by day 14. Similar patterns of labeling were seen with antibody $1 \mathrm{~B} 4$ (not shown). At day 12 through day 14 (Figs. 1C, 1D), labeling was more concentrated in the anterior stroma and subsequently became more widespread until a uniform distribution was seen throughout the full stromal thickness by day 18 . Counterstaining of corneal stromal cell nuclei with DAPI indicated a dis-
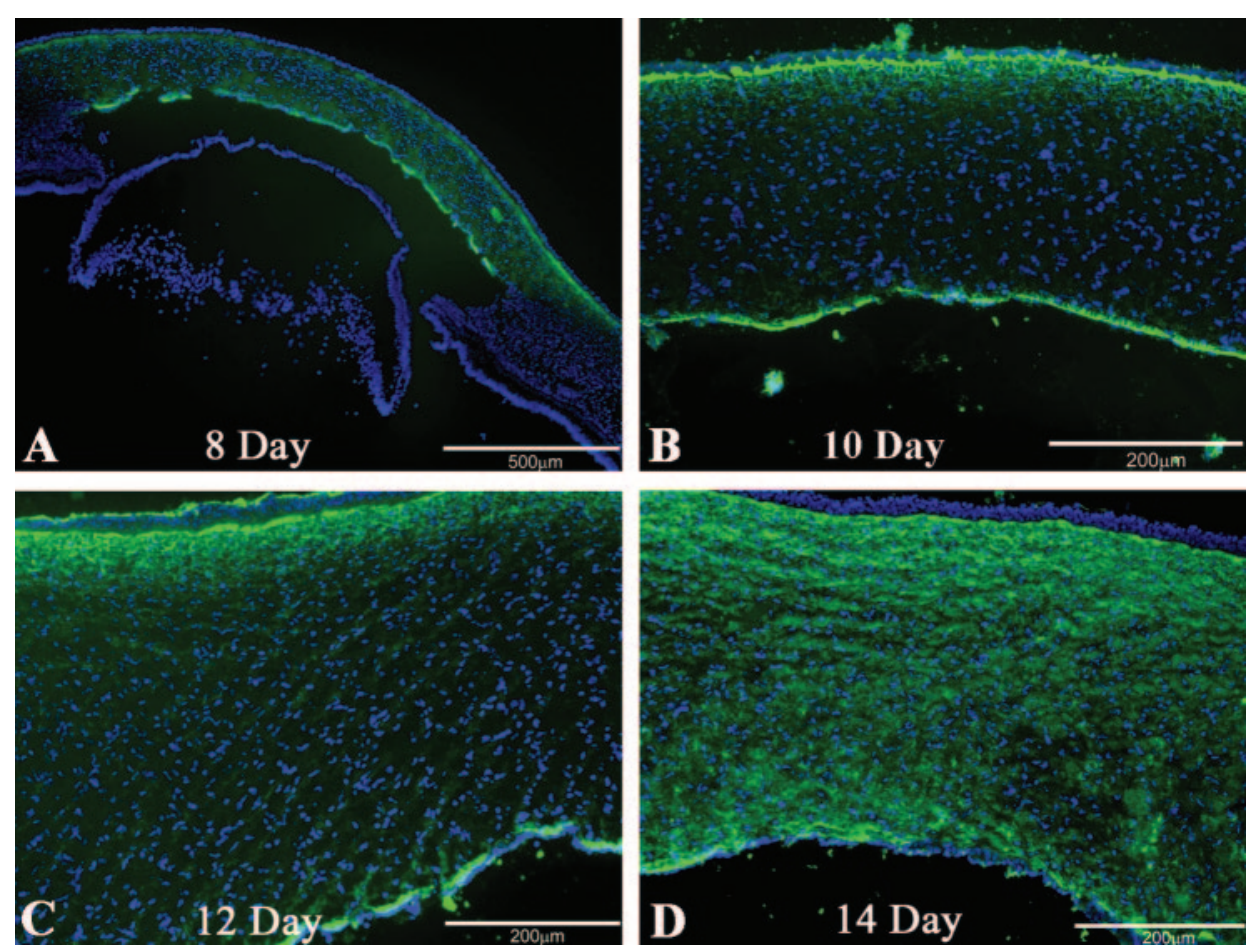

Figure 1. Localization of high-sulfated KS PGs in developing chick cornea by immunofluorescence with antibody 5D4 at 8 (A), 10 (B), 12 (C) and 14 (D) days of incubation. Alexa 488 (green) labels KS epitope with cell nuclei stained blue by DAPI. The anterior stroma stains more intensely than mid-posterior regions at days 12 and $14(\mathbf{C}, \mathbf{D})$. Scale bars, $500 \mu \mathrm{m}$ (A) and $200 \mu \mathrm{m}(\mathbf{B}-\mathbf{D})$. 
tinctive transformation from rounded to elongated morphology between day 14 and day 16, in accordance with previous observations by electron microscopy. ${ }^{19}$

Immunoelectron microscopy disclosed that antibody 5D4 consistently yielded a greater density of labeling with gold particles than antibody 1B4 (Figs. 2, 3). In both situations, however, sulfated KS epitopes were closely associated with bundles of collagen fibrils throughout days 8 to 18 , although at day 8 some collagen fibrils in the posterior stroma remained unlabeled with antibody 1B4 (Fig. 3A). Punctate immunofluorescence labeling with antibody 5D4 at day 8 was coincident with the presence of an early matrix consisting of scattered bundles of small groups of loosely associated collagen fibrils (Figs. 2A).

Collagen fibrils appeared closely associated with the cell membranes of presumptive keratocytes as tufts radiating from the cell surface (Fig. 3B) or as bundles contained in recesses of the plasma membrane (Fig. 3D). By day 10, a rudimentary lamellar arrangement of fibrils was already evident, together with increased density of labeling for sulfated KS, particularly with antibody 5D4 (Figs. 2B, 3B). Interestingly, individual stromal cells at day 14 often displayed multiple fibril bundles, each containing parallel skeins of fibrils with different orientations at separate locations along the membrane (Fig. 4). Sulfated KS showed a further increase in labeling density with both anti- bodies from day 12 through day 16 (Figs. 2C-E, 3C-E). This was accompanied by progressive consolidation of the stroma, as evidenced by increases in collagen fibril density and alignment and in the regularity of fibril spacing. By day 18, pericellular and interlamellar spaces devoid of collagen were not seen, and all fibrils were decorated with gold particles labeling 5D4 and 1B4 KS epitopes (Figs. 2F, 3F). Some sites along the plasma membranes of keratocytes were also heavily labeled with gold particles in both 5D4- and 1B4-treated sections (Figs. 2F, 3F).

\section{Discussion}

Numerous studies have been conducted of KS in the developing avian cornea since the early investigations of Anseth ${ }^{10}$ in 1961 and $\mathrm{Hart}^{11}$ in 1976 . The extent to which poly-N-lactosamine chains of KS are substituted with sulfate groups is likely to be critical in terms of the molecular interactions of KS PGs. Sulfation of KS would be expected to directly affect the molecular polarity of KS PGs, contributing to their waterbinding properties and their influence on tissue hydration ${ }^{20}$ and collagen interfibrillar spacing. Antibodies recognizing specific sulfation motifs on KS chains enable precise mapping by immunomicroscopy of the tissue location and extent of sulfation of KS PGs with a resolution down to the level of single
FIGURE 2. Ultrastructural localization of high-sulfated KS PGs in developing chick cornea with antibody 5D4 at 8 (A), 10 (B), 12 (C), 14 (D), 16 (E), and 18 (F) days of incubation. Collagen fibrils are decorated with gold particles indicating KS epitope with progressive increase in labeling as fibril density and alignment increase. Higher sulfated KS (5D4) epitope is more abundant than lower sulfated (1B4) epitope at all stages (see Fig. 3). Scale bars, $300 \mathrm{~nm}$.
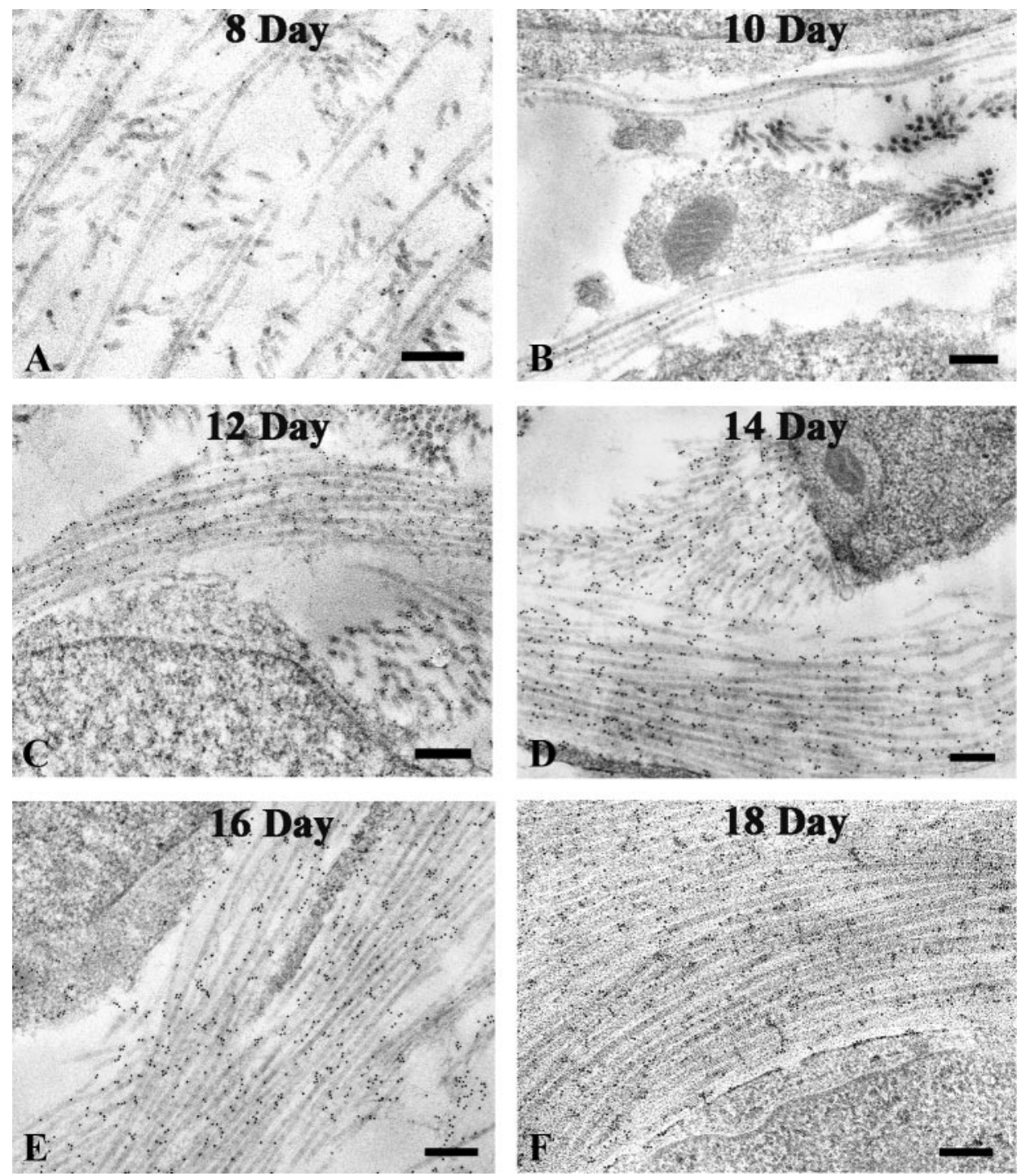

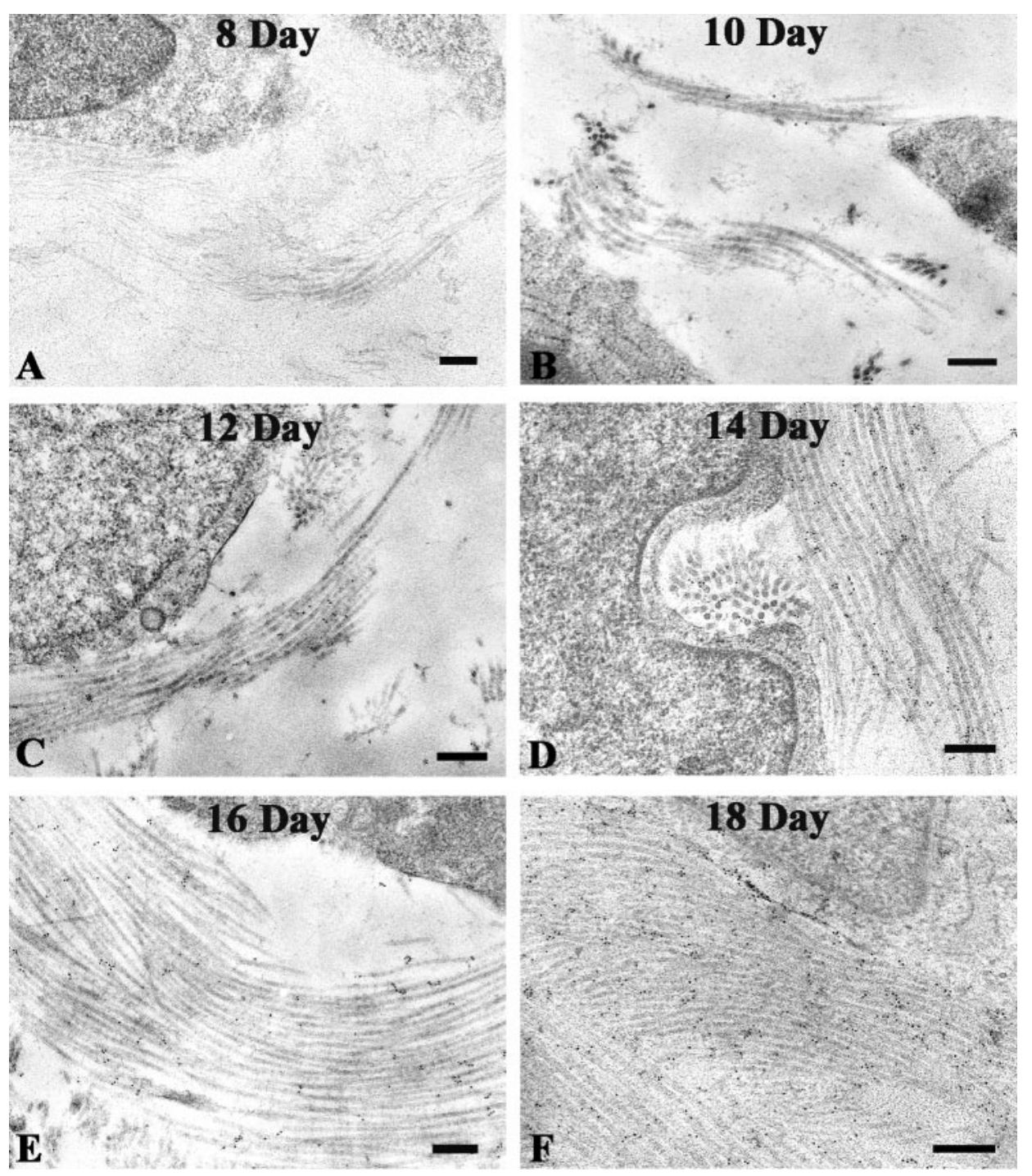

FIGURE 3. Ultrastructural localization of lower sulfated KS PGs in developing chick cornea with antibody $1 \mathrm{~B} 4$ at 8 (A), 10 (B), 12 (C), 14 (D), 16 (E), and 18 (F) days of incubation. Collagen fibrils are decorated with gold particles indicating KS epitope, with progressive increase in labeling as fibril density and alignment increase. Scale bars, $300 \mathrm{~nm}$. collagen fibrils. Antibody 5D4 immunoreactivity has a requirement for linear sequences of $N$-acetyllactosamine that are minimally pentasulfated and that we normally regard as highsulfated KS epitope, whereas antibody $1 \mathrm{~B} 4$ recognizes lesser sulfated oligosaccharide units. ${ }^{17,18}$ In the present study, immunodetection of low-sulfated KS epitope indicated low, but gradually increasing, levels throughout chick corneal development, whereas the high-sulfated epitope, identified by antibody 5D4, consistently appeared more abundant. 5D4 labeling showed that KS chains in embryonic chick corneal stroma express high-sulfated epitopes from an early stage, as reported previously. ${ }^{12,13}$ More intense labeling for high- and low-sulfated epitopes was present in anterior, compared with posterior, stroma at days 12 and 14, extending to uniform distribution by day 18 . This observation differs from earlier immunohistochemical studies in which increased posterior ${ }^{12}$ or uniform stromal localization of KS was reported. ${ }^{13}$ However, increased KS biosynthesis in the anterior stroma might be expected because this location coincides with the region of greatest cell density and collagen deposition at these developmental stages and may also be subject to low intracorneal oxygen tension, which some evidence suggests is a factor that predisposes toward KS biosynthesis. ${ }^{21,22}$ Weaker staining with antibody 5D4, as we observed in the posterior stroma, has previously been interpreted as undersulfation of $\mathrm{KS},{ }^{13}$ but this is perhaps less likely than the possibility that KS chains have not yet attained full length at this developmental stage, bearing in mind that current evidence suggests that sulfation of specific residues on $\mathrm{KS}$ poly- $N$-acetyllactosamine is linked to elongation of the KS chain. ${ }^{23-25}$

Positive immunolabeling with 1B4 and 5D4 antibodies represents collagen-bound KS PG rather than free KS GAG chains. KS chains are thought to be elaborated through the activities of glycosyl transferase enzymes and substituted onto core protein on the ribosomes, then substituted with sulfate groups by sulfotransferase enzymes in the Golgi compartment of the cell. Furthermore, high- and low-sulfate epitopes may be present within single KS chains. Studies on adult pig cornea suggested that chains display a distinct sulfation pattern, with a nonsulfated disaccharide at the reducing end close to the core protein linkage, next to a constant domain of monosulfated disaccharides and a variable number of disulfated disaccharides toward the nonreducing end of the molecule. ${ }^{26}$ The KS epitopes identified in our study could reside on chains linked to any of the three KS PG core proteins, lumican, keratocan, and mimecan, known to be present in the developing chick cornea, though at present we are unable to identify their specific core protein associations. However, we previously demonstrated by immunofluorescence microscopy keratocan distribution in anterior chick corneal stroma (Quantock AJ. IOVS 2006;47:ARVO E-Abstract 3005), which matches the distribution of the 5D4-labeled high-sulfated KS at early developmental stages we find in the 


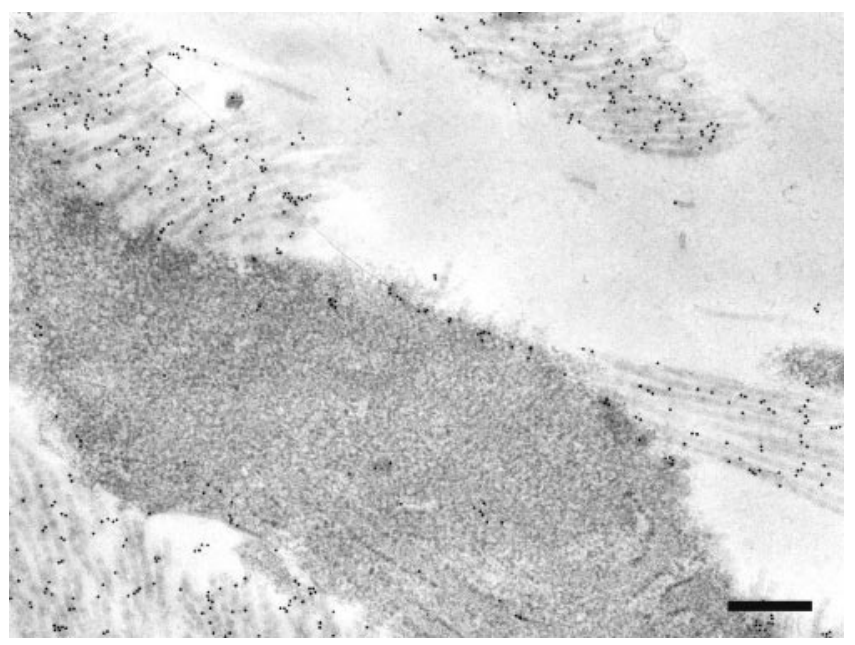

FIGURE 4. Ultrastructural localization of sulfated KS in developing chick cornea. A fibroblast at day 14 is associated with several 5D4labeled (high-sulfated KS) collagen bundles with fibrils at various orientations. Scale bar, $300 \mathrm{~nm}$.

present study. Although lumican is known to be the major corneal KS PG in chick, keratocan may carry more KS chains, as is the case in bovine cornea. ${ }^{27}$ Therefore, keratocan might be a more likely candidate for KS sulfation, particularly in early stages of development. Because keratocan is regulated transcriptionally by lumican, ${ }^{28}$ lumican would also be expected to be present in the developing matrix at an early stage. Indeed, elegant studies on corneal KS PG core proteins by Dunlevy et al. ${ }^{15}$ indicated that lumican mRNA expression remained high throughout chick corneal development. Even so, the protein was found to be secreted in an unsulfated form until day 15 of development, with sulfated lumican KS PG appearing only thereafter as tissue transparency increased. ${ }^{29}$ Our observations of increasing levels of detectable high-sulfated KS epitope in the chick stroma from day 8 , together with these earlier data, invite speculation that keratocan may be the main carrier protein for sulfated KS chains in the earlier stages of corneal development. Keratocan may therefore fulfill an important role in the initial stages of collagen fibril organization and orientation of presumptive lamellae, whereas lumican supersedes when tighter regulation of stromal hydration and tissue compaction is required to produce a transparent matrix.

Immunoelectron microscopy demonstrated that extended sequences of high-sulfated disaccharides on KS appear simultaneously with extracellular accretion of collagen fibrils. From day 8 in chick cornea, both types of sulfated KS epitopes, the high-sulfate epitope in particular, appeared in greatest density where collagen fibril deposition was most advanced. Moreover, KS immunolabeling was invariably associated with collagen fibrils at the surfaces of stromal cells. No isolated label was located in collagen-free spaces of the developing corneal matrix. Collagen fibrils assembling at cell surfaces are, therefore, already endowed with sulfated KS PG as they emerge into the matrix and aggregate first as fibril bundles, with collagen fibrils apparently equally spaced, and then as lamellae. ${ }^{30}$ The early appearance of sulfated corneal KS is consistent with its potential involvement in matrix organization at the beginning of fibrillogenesis in the secondary stroma. In day 14 and later corneas, we frequently observed dense, aligned skeins of fibrils, which we presumed to be rudimentary lamellae, tightly apposed to involuted membranes of stromal cells. Recent studies on fibrillogenesis in embryonic tendon, where- unlike in cornea-fibrils are deposited in parallel register along a single geometric axis, have shown that fibrils form within the cells, inside membrane-bound inclusions termed Golgi to plasma membrane carriers. ${ }^{31}$ Groups of fibrils are eventually conducted to the extracellular space by "fibripositor" protrusions of the cell membrane, which adopt alignment along the cell and tendon axis in association with the actin cytoskeleton. ${ }^{32}$ Compartmentalized intracellular assembly of matrix components would conceivably offer significant advantages of a controlled milieu for interactions between collagen and PGs, facilitating the establishment of appropriate geometric relationships before insertion into the extracellular matrix. However, analogous structures have not yet been described in cornea, where the requirement to deposit fibrils in more than one orientation would seem inherently more complex than uniaxial fibrillogenesis in tendon. Migration of neural crest-derived cells into the developing embryonic cornea is well documented, ${ }^{1,2}$ yet the cellular mechanisms and sequences of biosynthesis of the extracellular lamellar collagen architecture are still unknown. Extrusion of fibrils may occur as cells migrate, with direct lines of cell movement subsequently determining lamellar orientation. The possibility that migrating cells may simultaneously release fibrils assuming different matrix orientations implies close cellular control over the deposition of rudimentary lamellae, and some of our observations on 14-day embryos occasionally indicated up to three apparently separate orientations of KS-labeled fibril bundles associated with individual cells. This complex process may preclude bundle assembly inside the cell, as in tendons, and may necessitate the accretion of components in membrane channels at the cell surface. Clearly, three-dimensional imaging techniques will be required to explore more fully the potential for simultaneous deposition of multiple lamellae by corneal cells. We postulate that coordinated synthesis of sulfated KS PG with collagen fibrillogenesis is essential for functional assembly of rudimentary lamellae in corneal development.

\section{References}

1. Coulombre AJ, Coulombre JL. The development of the structural and optical properties of the cornea. In: Smelser GK, ed. The Structure of the Eye. New York: Academic Press; 1961;405- 420.

2. Hay ED, Revel J-P. Fine structure of the developing avian cornea. In: Wolsky A, Chen PS, eds. Monographs in Developmental Biology. Vol. 1. Basel, Switzerland: S. Karger; 1969;1-144.

3. Trelstad RL, Coulombre AJ. Morphogenesis of the collagenous stroma in the chick cornea. J Cell Biol. 1971;50:840-858.

4. Hay ED. Development of the vertebrate cornea. Int Rev Cytol. 1980;63:263-321.

5. Linsenmayer TF, Fitch JM, Gordon MK, et al. Development and roles of collagenous matrices in the embryonic avian cornea. Prog Retin Eye Res. 1998;17:231-265.

6. Maurice DM. The structure and transparency of the cornea. J Physiol (Lond). 1957;186:263-286.

7. Benedek GB. Theory of transparency of the eye. Appl Opt. 1971; 10:459-473.

8. Farrell RA. Corneal transparency. In: Albert DM, Jacobiec SA, eds. Principles and Practice of Opbthalmology. Philadelphia: WB Saunders; 1994.

9. Birk DE, Trelstad RL. Extracellular compartments in matrix morphogenesis: collagen fibril, bundle, and lamellar formation by corneal fibroblasts. J Cell Biol. 1984;99:2024-2033.

10. Anseth A. Glycosaminoglycans in the developing corneal stroma. Exp Eye Res. 1961;1:116-121.

11. Hart GW. Biosynthesis of glycosaminoglycans during corneal development. J Biol Chem. 1976;251:6513-6521.

12. Funderburgh JL, Caterson B, Conrad GW. Keratan sulfate proteoglycan during embryonic development of the chicken cornea. Dev Biol. 1986;116:267-277.

13. Takahashi I, Nakamura Y, Hamada Y, Nakazawa K. Immunohistochemical analysis of proteoglycan biosynthesis during early development of the chicken cornea. J Biochem. 1999;126:804-814. 
14. Nakazawa K, Suzuki S, Wada K, Nakazawa K. Proteoglycan synthesis by corneal explants from developing embryonic chicken. J Biochem. 1995;117:707-718.

15. Dunlevy JR, Beales MP, Berryhill BL, Cornuet PK, Hassell JR. Expression of the keratan sulfate proteoglycans lumican, keratocan and osteoglycin/mimecan during chick corneal development. Exp Eye Res. 2000;70:349-362.

16. Zhang Y, Conrad AH, Tasheva ES, et al. Detection and quantification of sulfated disaccharides from keratan sulfate and chondroitin/dermatan sulfate during chick corneal development by ES-MS/MS. Invest Ophthalmol Vis Sci. 2000;46:1604-1614.

17. Caterson B, Christner JE, Baker JR, Couchman JR. Production and characterization of monoclonal antibodies directed against connective tissue proteoglycans. Fed Proc. 1985;44:386-393.

18. Mehmet H, Scudder P, Tang PW, Hounsell EF, Caterson B, Feizi T. The antigenic determinants recognized by three monoclonal antibodies to keratan sulphate involve sulphated hepta- or larger oligosaccharides of the poly(N-acetyllactosamine) series. Eur J Biochem. 1986;157:385-391.

19. Connon CJ, Siegler V, Meek KM, et al. Proteoglycan alterations and collagen reorganisation in the secondary avian cornea during development. Ophthal Res. 2003;35:177-184.

20. Bettelheim FA, Plessy B. The hydration of proteoglycans of bovine cornea. Biochim Biophys Acta. 1975;383:203-214.

21. Cintron C, Covington HI, Kublin CL, Gregory JD, Damle SP. Developmental aspects of keratan sulphate. In: Greiling $\mathrm{H}$, Scott JE, eds. Keratan Sulphate: Chemistry, Biology and Chemical Patbology. London: The Biochemical Society; 1989:158-167.

22. Scott JE, Haigh M. Keratan sulphate and the ultrastructure of cornea and cartilage: a "stand-in" for chondroitin sulphate in conditions of oxygen lack? J Anat. 1988;158:95-108.

23. Keller R, Stuhlsatz HW, Greiling H. Sulphation, chain elongation and chain termination in keratan sulphate biosynthesis. In: Greil- ing H, Scott JE, eds. Keratan Sulphate: Chemistry, Biology and Chemical Patbology. London: The Biochemical Society; 1989:3952.

24. Akama TO, Nakayama J, Nishida K, et al. Human corneal GlcNac 6-O-sulfotransferase and mouse intestinal GlcNac 6-O-sulfotransferase both produce keratan sulfate. J Biol Chem. 2001;276: 16271-16278.

25. Akama TO, Misra AK, Hindsgaul O, Fukuda MN. Enzymatic synthesis in vitro of the disulfated disaccharide unit of keratan sulfate. J Biol Chem. 2002;277:42505-42513.

26. Oeben M, Keller R, Stuhlsatz W, Greiling H. Constant and variable domains of different disaccharide structure in corneal keratan sulphate chains. Biochem J. 1987;248:85-93

27. Funderburgh JL, Funderburgh ML, Mann MM, Conrad GW. Unique glycosylation of three keratan sulfate proteoglycan isoforms. $\mathrm{J} \mathrm{Biol}$ Chem. 1991;266:14226-14231.

28. Carlson EC, Liu CY, Chikama TI, et al. Keratocan, a cornea-specific keratan sulphate proteoglycan, is regulated by lumican. J Biol Chem. 2005;280:25541-25547.

29. Cornuet PK, Blochberger TC, Hassell JR. Molecular polymorphism of lumican during corneal development. Invest Opbthalmol Vis Sci. $1994 ; 35: 870-877$.

30. Chakravarti S, Zhang G, Chervoneva I, Roberts L, Birk DE. Collagen fibril assembly during postnatal development and dysfunctional regulation in the lumican-deficient murine cornea. Dev Dyn. 2006; 235:2493-2506.

31. Canty EG, Lu YH, Meadows RS, Shaw MK, Holmes DF, Kadler KE. Coalignment of plasma membrane channels and protrusions (fibripositors) specifies the parallelism of tendon. J Cell Biol. 2004; 165:553-563

32. Canty EG, Starborg T, Lu Y, et al. Actin filaments are required for fibripositor-mediated collagen fibril alignment in tendon. $J$ Biol Chem. 2006;281:38592-38598. 